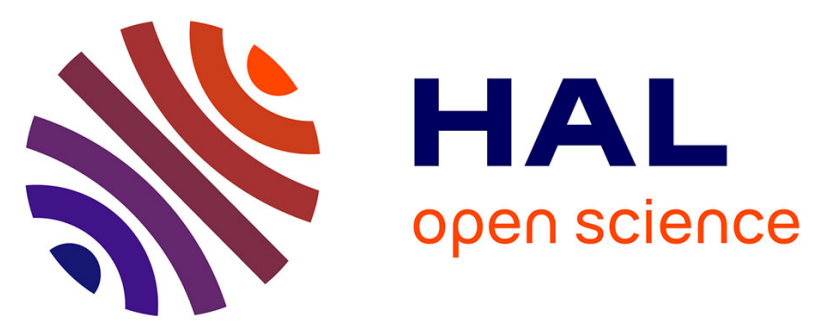

\title{
A discrete modeling-based constitutive relation for infilled rock joints
}

Jérôme Duriez, F. Darve, F.-V. Donzé

\section{To cite this version:}

Jérôme Duriez, F. Darve, F.-V. Donzé. A discrete modeling-based constitutive relation for infilled rock joints. International Journal of Rock Mechanics and Mining Sciences, 2011, 48 (3), pp.458-468. 10.1016/j.ijrmms.2010.09.008 . hal-01903579

\section{HAL Id: hal-01903579 \\ https://hal.science/hal-01903579}

Submitted on 24 Oct 2018

HAL is a multi-disciplinary open access archive for the deposit and dissemination of scientific research documents, whether they are published or not. The documents may come from teaching and research institutions in France or abroad, or from public or private research centers.
L'archive ouverte pluridisciplinaire HAL, est destinée au dépôt et à la diffusion de documents scientifiques de niveau recherche, publiés ou non, émanant des établissements d'enseignement et de recherche français ou étrangers, des laboratoires publics ou privés. 


\title{
A discrete modeling-based constitutive relation for infilled rock joints
}

\author{
J.Duriez ${ }^{\mathrm{a}, *}$, F.Darve ${ }^{\mathrm{a}}$, F.-V.Donzéa ${ }^{\mathrm{a}, \mathrm{b}}$ \\ ${ }^{a}$ Laboratoire Sols, Solides, Structures et Risques, UMR5521, Université Joseph Fourier, Grenoble-INP, \\ Grenoble Universités, Domaine Universitaire, BP 53, 38041 Grenoble Cedex 9, France \\ ${ }^{b}$ Earth Science and Resource Engineering, CSIRO, Queensland Centre for Advanced \\ Technologies, 1 Technology Court, Pullenvale, 4069, Australia
}

\begin{abstract}
An incrementally nonlinear constitutive relation is formulated to describe the mechanical behaviour of infilled rock joints. This relation is calibrated using a discrete element model, which is validated by experimental data. Since the phenomenological relation fully combines the normal and tangential directions of a rock joint, it can reproduce rock joint features as the dilatancy process and the contribution of compression on tangential stress. To take into account the hardening of the material, the influence of the previous shear loading history on the mechanical response of the rock joint is considered. Finally, the performance of the constitutive relation is verified by showing the good agreement between the responses predicted by the relation and those obtained by the discrete model for different loading paths.
\end{abstract}

Rock joints present a wide variety of features that influence their mechanical behaviour. Among them is the presence and the type of filling material. Filled discontinuities can occur after erosion of adjoining blocks, due to relative shearing: crushing of asperities will then give birth to a gouge. Fillers can also have been deposited, by water flows for example. Many previous studies investigating different situations have emphasized that the presence of this filling material modifies the shear strength of the rock joint.

Papaliangas et al. [1] presented the experimental results of direct shear tests on model discontinuities (casted from a sandstone sample) filled with various thicknesses of pulverised fuel ash: a non-cohesive, fine-grained material. Obviously, increasing fill thickness, starting from a clean discontinuity, led to decreasing values of peak or residual tangential stresses. Decreases of the peak shear stress by a ratio between 2 and 3 have been shown.

The same type of results were observed by Pereira [2] on sandstone discontinuities filled or not with a river sand. After circular shear tests, the peak friction coefficient was found to decrease from $28^{\circ}$ in a clean case to even $10.2^{\circ}$. A similar study (Kutter \& Raunternberg [3]), also on sandstone discontinuties with sand, revealed the same trend.

*Corresponding author

Preprint submitted to Elsevier 
Other studies in the framework of cohesive infill materials presented the same results. Lama [4], who studied models of sandstone sample filled with kaolin, also observed a decrease of the peak shear strength depending on the thickness of the filling material. Indraratna and Haque [5] drew the same conclusions after experiments on artificial plaster discontinuities containing bentonite.

A last example of such results, within a different framework, can be found in the work of Boulon et al. [6]. These authors studied natural calcite-healed discontinuities of granodiorite. And the presence of calcite infill material led also to decreasing shear strength.

Because of this lower shear strength, filled discontinuities are critical in rock mass stability studies and they must be considered. They are therefore the subject of this paper focusing on their mechanical behaviour. The aim is hence to define a new constitutive relation able to simulate this behaviour.

Several constitutive laws have already been proposed in the literature. Historically let us mention Goodman [7] who defined three mechanical characteristics to represent the mechanical behaviour of rock joints: a constant normal stiffness, which links the normal stress to the normal displacement across the joint, a constant tangential stiffness, which links the tangential stress to the tangential displacement along the joint, and a maximum value for the tangential stress. This gives an elastic-plastic constitutive relation with a diagonal stiffness matrix. As Goodman said, because of the lack of nondiagonal terms (which could combine tangential displacement with normal stress, for example) in the matrix, it is not possible to take into account the dilatancy of rock joints that is often measured (as in [1] or [2] for infilled discontinuities). Bandis et al.[8] reconsidered only these two normal and tangential stiffnesses. The model was nevertheless improved as for the normal direction a nonconstant normal stiffness was considered, corresponding to the nonlinear functions $\sigma(u)$ that these authors measured.

A constitutive relation with a full stiffness matrix was proposed by Leichnitz [9] and by Saeb and Amadei [10], both with non-constant coefficients.

But, linked with their (incrementally) linear nature, all these relations can describe only elastic behaviours - in [8] the inelasticity in normal direction is studied, but not in the tangential direction - , whereas rock joints often exhibit inelastic deformational behaviour.

Using the more conceptual elasto-plastic framework, as Plesha did [11], allows one to define inelastic models. In the corresponding study, the displacement increment is divided into two elastic and plastic parts. The elastic one is computed through a diagonal matrix whose coefficients are constant; for the plastic part, a yield function $F$ and a plastic potential $G$ are defined and used. The corresponding flow rule is nonassociated $(G$ different from $F$ ) because this paper emphasises that associated models can hardly ever satisfactorily simulate dilatancy.

Unfortunately, flow rules are very difficult to obtain experimentally. This elastoplastic framework will therefore not be used here. Instead, the present study is based on Darve's incrementally nonlinear formalism [12], which has been shown to be efficient for soils [13] and which will be presented in first section of this paper. This formalism will allow the constitutive relation to take into account the inelasticity of 
rock joints, to be as general as possible by not referring to any particular boundary conditions (contrary to [7] or [8]) and to combine, as nature does, normal and tangential directions. As for rock joints, it is very difficult to obtain a consistent experimental database, we have chosen to built first a numerical database using the discrete element method (DEM, [14]); this method has recently been used in the rock joint context: see $[15,16,17]$. The presence of an infill material in the discontinuities considered here makes this a good choice. This material will be considered a granular material (as in [1] or [2]), and the DEM has shown repeatedly its ability to describe the complex behaviour of granular media both qualitatively and quantitatively [18, 19, 20, 21, 22].

After a presentation of the construction of the constitutive relation, the second part of the paper will present the discrete element model used. Then the numerical results, with few available experimental data, will be used to calibrate the constitutive relation. Finally this relation will be validated through comparisons with the same numerical model, within a general rock joint loading framework.

\section{The constitutive relation for rock joints}

By defining $\sigma$ as the normal stress acting at the interface, $\tau$ as the tangential one, $u$ as the amount of normal relative displacement between the blocks and $\gamma$ the tangential one, the rate-independent behaviour of a rock joint is described incrementally by the following expressions:

$$
\left\{\begin{array}{l}
d \tau=f_{h}(d \gamma, d u) \\
d \sigma=g_{h}(d \gamma, d u)
\end{array}\right.
$$

where the vectorial functions $f$ and $g$ depend on state variables and memory parameters denoted by $h$.

Because of the rate-independency condition, $f$ and $g$ have to be invariant with respect to rates, which implies:

$$
\forall \lambda>0:\left\{\begin{array}{l}
\lambda d \tau=f_{h}(\lambda d \gamma, \lambda d u) \\
\lambda d \sigma=g_{h}(\lambda d \gamma, \lambda d u)
\end{array}\right.
$$

Thus, $\forall \lambda>0$ :

$$
\begin{cases}f_{h}(\lambda d \gamma, \lambda d u)= & \lambda f_{h}(d \gamma, d u) \\ g_{h}(\lambda d \gamma, \lambda d u)= & \lambda g_{h}(d \gamma, d u)\end{cases}
$$

which is the definition of homegeneous vectorial functions of degree 1. According to Euler's identity for homogeneous functions, it turns out that:

$$
\left\{\begin{array}{l}
f_{h}(d \gamma, d u)=\frac{\partial f_{h}}{\partial(d \gamma)} d \gamma+\frac{\partial f_{h}}{\partial(d u)} d u \\
g_{h}(d \gamma, d u)=\frac{\partial g_{h}}{\partial(d \gamma)} d \gamma+\frac{\partial g_{h}}{\partial(d u)} d u
\end{array}\right.
$$


or in an equivalent way:

$$
\left(\begin{array}{c}
d \tau \\
d \sigma
\end{array}\right)=\left(\begin{array}{c}
f_{h}(d \gamma, d u) \\
g_{h}(d \gamma, d u)
\end{array}\right)=\left(\begin{array}{cc}
\frac{\partial f_{h}}{\partial(d \gamma)} & \frac{\partial f_{h}}{\partial(d u)} \\
\frac{\partial g_{h}}{\partial(d \gamma)} & \frac{\partial g_{h}}{\partial(d u)}
\end{array}\right)\left(\begin{array}{c}
d \gamma \\
d u
\end{array}\right)=M_{h}(d \gamma, d u)\left(\begin{array}{c}
d \gamma \\
d u
\end{array}\right)
$$

This proves the existence of the elasto-plastic matrix $M_{h}$. Since the partial derivatives of homegeneous functions of degree 1 are homogeneous functions of degree 0 , the matrix $M_{h}$ depends only on the direction of the vector $\left(\begin{array}{c}d \gamma \\ d u\end{array}\right)$ and not on its norm. Thus:

$$
M_{h}(d \gamma, d u)=M_{h}\left(\frac{d \gamma}{\sqrt{d \gamma^{2}+d u^{2}}}, \frac{d \gamma}{\sqrt{d \gamma^{2}+d u^{2}}}\right)
$$

Let us now consider the Taylor's series expansion for $M_{h}$. This gives:

$$
M_{h}=M_{h}^{1}+\frac{1}{\sqrt{d \gamma^{2}+d u^{2}}} M_{h}^{2}\left(\begin{array}{c}
d \gamma \\
d u
\end{array}\right)+\cdots
$$

where $M_{h}^{1}$ is a rank 2 tensor and $M_{h}^{2}$ rank 3 .

Thus the rate-independent constitutive relation of rock joints can be written under the following form:

$$
\left(\begin{array}{c}
d \tau \\
d \sigma
\end{array}\right)=M_{h}^{1}\left(\begin{array}{c}
d \gamma \\
d u
\end{array}\right)+\frac{1}{\sqrt{d \gamma^{2}+d u^{2}}} M_{h}^{2}\left(\begin{array}{c}
d \gamma \\
d u
\end{array}\right)\left(\begin{array}{c}
d \gamma \\
d u
\end{array}\right)+\cdots
$$

The general expression of the incrementally nonlinear second-order constitutive relation for rock joints is then given by:

$$
\left(\begin{array}{c}
d \tau \\
d \sigma
\end{array}\right)=M_{h}^{1}\left(\begin{array}{c}
d \gamma \\
d u
\end{array}\right)+\frac{1}{\sqrt{d \gamma^{2}+d u^{2}}} N_{h}^{2}\left(\begin{array}{c}
d \gamma^{2} \\
d u^{2}
\end{array}\right)
$$

where the crossed terms $d u d \gamma$ are not considered.

The matrices $M_{h}^{1}$ and $N_{h}^{2}$ are defined through two calibration paths:

- the first path is controlled by the following conditions ( $d u=0 ; d \gamma$ given constant), which is a constant normal displacement (CND) path. This is indeed a constant volume path.

- the second calibration path is defined by ( $d u$ given constant; $d \gamma=0)$, which is called the constant tangential displacement (CTD) path. This corresponds to an odometric compression because possibility of lateral deformation is cancelled.

Considering the curves $\sigma(\gamma)$ and $\tau(\gamma)$ from the CND path and the curves $\sigma(u)$ and $\tau(u)$ from the CTD path, the following moduli, depending on the state variables and memory parameters, can be defined: 


$$
\begin{array}{rlrl}
G_{\gamma}^{+} & =\frac{\partial \tau}{\partial \gamma}_{u, d \gamma>0} & G_{\gamma}^{-} & =\frac{\partial \tau}{\partial \gamma}_{u, d \gamma<0} \\
N_{\gamma}^{+}=\frac{\partial \sigma}{\partial \gamma}_{u, d \gamma>0} & N_{\gamma}^{-} & =\frac{\partial \sigma}{\partial \gamma}_{u, d \gamma<0} \\
G_{u}^{+} & =\frac{\partial \tau}{\partial u} & G_{u, d u>0}^{-} & =\frac{\partial \tau}{\partial u}_{\gamma, d u<0} \\
N_{u}^{+} & =\frac{\partial \sigma}{\partial u}_{\gamma, d u>0} & N_{u}^{-} & =\frac{\partial \sigma}{\partial u}_{\gamma, d u<0}
\end{array}
$$

Let us now introduce the matrices $P^{+}$and $P^{-}$defined by:

$$
P^{+}=\left(\begin{array}{cc}
G_{\gamma}^{+} & G_{u}^{+} \\
N_{\gamma}^{+} & N_{u}^{+}
\end{array}\right) \quad P^{-}=\left(\begin{array}{cc}
G_{\gamma}^{-} & G_{u}^{-} \\
N_{\gamma}^{-} & N_{u}^{-}
\end{array}\right)
$$

which contribute to the computation of $M_{h}^{1}$ and $N_{h}^{2}$ by identifying the incrementally nonlinear constitutive second-order relation on the calibration paths through matrices $P^{+}$and $P^{-}$:

$$
\left\{\begin{array}{l}
M_{h}^{1}=\frac{1}{2}\left(P^{+}+P^{-}\right) \\
N_{h}^{2}=\frac{1}{2}\left(P^{+}-P^{-}\right)
\end{array}\right.
$$

Thus, the constitutive relation becomes:

$$
\left(\begin{array}{c}
d \tau \\
d \sigma
\end{array}\right)=\frac{1}{2}\left(P^{+}+P^{-}\right)\left(\begin{array}{c}
d \gamma \\
d u
\end{array}\right)+\frac{1}{2 \sqrt{d u^{2}+d \gamma^{2}}}\left(P^{+}-P^{-}\right)\left(\begin{array}{c}
d \gamma^{2} \\
d u^{2}
\end{array}\right)
$$

To use this relation, only the values of the moduli need to be known. Unfortunately, if experimental compressions of rock joints can generally be considered œedeometric, there is clearly a lack of experimental data for the other calibration path: the constant normal displacement path. In the case of rock joints, which very often present dilatancy during shearing, the stiffnesses of the experimental device needed to cancel all normal relative displacements are indeed very difficult to obtain. We therefore decided to create a numerical model which could represent the behaviour of a rock joint and to use it to simulate these calibration paths.

\section{The discrete element model}

The discrete element method (DEM) [14, 21] considers a set of different elements (or particles) interacting with each other whose movements obey the second law of Newton. Using this numerical method, some authors have already simulated rock joints: see $[15,16,17]$. Starting from a set of bonded particles which represent the intact rock, the joints are simulated in these studies by suppressing the contact bond strengths for all contacts located across a line which represent the rock joint. These simulations are adapted for rock joints that correspond to rough blocks sliding directly over each other. On the other hand, the rock joints considered here present a filling material, which corresponds to a vanishing Joint Roughness Coefficient (JRC, see [23] for example). Consequently, the construction presented here will be slightly different. 
However, as the discrete description has now proved its efficiency in simulating granular geomaterials such as soils (see [24, 25], for example), such discrete simulations can also be used to study the rock joints filled with gouge: we already stated that this filler is considered a granular material. Thus, the numerical model is developed with YADE [26, 27], open source discrete element software. The joint itself is simulated, as the spacing between two adjacent blocks which bounds the filling material. The interactions between the elements of the model are presented, then presentations of the model of the rock joint itself, and the simulations performed.

\subsection{Contact laws used}

Considering two spheres, $A$ and $B$, in contact, whose centres are, respectively, $C_{A}$ and $C_{B}$, and whose radii are $R_{A}$ and $R_{B}$, a penetration depth is defined for this interaction by:

$$
u_{n}=R_{A}+R_{B}-\left\|\overrightarrow{C_{A} C_{B}}\right\|
$$

\subsubsection{Normal contact force}

It can be considered that rock joints nearly always present an inelastic behaviour in compression, with a greater stiffness in unloading than in loading (see [7]). This feature is introduced in the numerical model at the scale of the interaction between spheres by computing the value of the normal contact force at each time step " $i$ " as follows:

$$
\begin{gathered}
F_{n}^{i}=\left\{\begin{array}{cc}
k_{n} u_{n}^{i} & \text { if } u_{n}^{i} \geqslant \max _{k, k \in \llbracket 1 ; i-1 \rrbracket}\left(u_{n}^{k}\right) \\
F_{n}^{i-1}+K k_{n}\left(u_{n}^{i}-u_{n}^{i-1}\right) & \text { if } u_{n}^{i}<\max _{k, k \in \llbracket 1 ; i-1 \rrbracket}\left(u_{n}^{k}\right)
\end{array}\right. \\
\text { where } k_{n}=\frac{2 Y_{A} R_{A} Y_{B} R_{B}}{Y_{A} R_{A}+Y_{B} R_{B}}
\end{gathered}
$$

The " $Y$ " are parameters of the numerical model and control the interaction stiffness $k_{n}$. $K$ is another parameter setting the difference in stiffnesses between loading and unloading $(K>1)$.

\subsubsection{Tangential contact force}

This force is computed incrementally. Starting from $\vec{F}_{t}=\overrightarrow{0}$ at the creation of the interaction, $\vec{F}_{t}$ is incremented at each time step indexed " $i$ " as follows:

$$
\begin{gathered}
\overrightarrow{F_{t}^{i}}=\overrightarrow{F_{t}^{i-1}}+k_{s} \overrightarrow{v_{r e l}^{M}(A / B)^{i}} \times d t \\
\text { where } k_{s}=\frac{2 Y_{A} P_{A} R_{A} Y_{B} P_{B} R_{B}}{Y_{A} P_{A} R_{A}+Y_{B} P_{B} R_{B}}
\end{gathered}
$$

In this equation, $\overrightarrow{v_{r e l}^{M}(A / B)}$ is the relative velocity between the two interacting discrete elements at the contact point $M$ and $d t$ the value of the time step used for the simulations. Parameters $P_{A}$ and $P_{B}$ control the tangential stiffness $k_{s}$. The value of the tangential force is limited by a plastic threshold, depending on the normal force in a 
classical Coulombian way. Thus:

$$
\left(\left\|\overrightarrow{F_{t}}\right\| \geqslant\left\|\overrightarrow{F_{n}}\right\| \tan \left(\phi_{\text {micro }}\right)\right) \Rightarrow\left(\overrightarrow{F_{t}}=\frac{\vec{F}_{t}}{\left\|\vec{F}_{t}\right\|}\left\|\overrightarrow{F_{n}}\right\| \tan \left(\phi_{\text {micro }}\right)\right)
$$

\subsubsection{Computation of a torque transmitted between the particles}

In this numerical model, as in many others using the discrete element method, the discrete elements used are spherical for practical reasons (this shape speeds up contact detection, for example). But because spheres roll easily over each other, it is now recognized that this leads to underestimating the shear strength of generally non-spherical granular materials (see [24]) that have to be simulated. A choice (made by the same authors [24]) is to cancel any rotation of the spheres in the numerical model. However some experimental studies on filled discontinuities consider infill material as more or less spherical ones: for example Papaliangas et al. [1] who focused on pulverised fuel ash, and Pereira [2] who investigated river sand. These authors both emphasized the existence of rolling infill grains. Thus, in order to approach reality, we made here the intermediate choice of using spherical particles, but with a transfer moment law that will induce resistant torques; so that particle rolling is reduced but not cancelled. The formulation of this moment transfer law is the one introduced by Iwashita and Oda [28] in two dimensions and developed in three dimensions by Plassiard [29].

A rolling angular vector $\vec{\theta}_{r}$ is defined for the interaction between spheres $A$ and $B$. This rolling vector represents the relative changes in orientation between the two particles. In a transfer moment law, these changes should induce a resistant couple $\vec{M}_{r}$. This couple is computed from an elastic-plastic point of view, such that:

$$
\begin{gathered}
\qquad \vec{M}_{r}=\left\{\begin{array}{cc}
k_{r}{\overrightarrow{\theta_{r}}}_{r} & \text { if }\left\|k_{r} \vec{\theta}_{r}\right\|<\left\|\vec{M}_{r}\right\|_{\text {lim }} \\
\left\|\vec{M}_{r}\right\|_{\text {lim }} \frac{\vec{\theta}_{r}}{\left\|\vec{\theta}_{r}\right\|} & \text { else }
\end{array}\right. \\
\text { where } k_{r}=\beta_{r}\left(\frac{R_{A}+R_{B}}{2}\right)^{2} k_{s} \text { and }\left\|\vec{M}_{r}\right\|_{l i m}=\eta_{r}\left\|\vec{F}_{n}\right\| \frac{R_{A}+R_{B}}{2}
\end{gathered}
$$

Increasing the value of the numerical parameter $\beta_{r}$ leads to greater stiffness in rotation, and increasing the value of the numerical parameter $\eta_{r}$ induces a higher value of the couple's plastic threshold. All the values of the numerical parameters used in the model are presented in Table 1 .

Table 1: Micro-parameters of the numerical model

\begin{tabular}{|c|c|c|c|c|c|}
\hline$Y(\mathrm{~Pa})$ & $P$ & $\phi_{\text {micro }}\left({ }^{\circ}\right)$ & $K=\frac{k_{n}(\text { unload })}{k_{n}(\text { load })}$ & $\beta_{r}$ & $\eta_{r}$ \\
\hline $4 e 9$ & 0.04 & 37 & 4 & 0.12 & 1 \\
\hline
\end{tabular}

Once the contact forces are applied to each element, Newton's law is considered for each spherical element. To ensure convergence towards equilibrium states a damping 


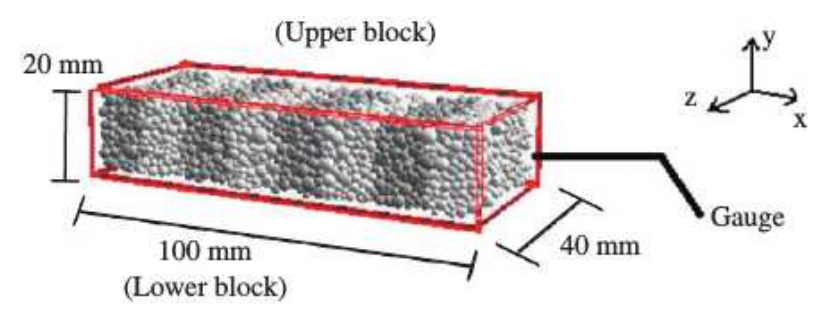

Figure 1: Geometry of the numerical model of a rock joint, with the frame of the axes

term is added to the sum of acting forces $\Sigma \vec{F}$. It is a nonviscous damping [30], $\vec{F}^{a}$ defined as:

$$
\vec{F}^{a}=-D \times \operatorname{sign}\left(\Sigma \vec{F}^{(t)} \cdot\left(\vec{v}^{(t)}+\frac{d t}{2} \vec{a}^{(t)}\right)\right)
$$

The advantage of this non-viscous damping is to increase the convergence speed, without disturbing simulations which would imply steady flows. $D$ is chosen equal to 0.2 . It is verified that this value does not modify the results.

Velocities and positions are finally updated through a second-order finite difference scheme.

\subsection{Numerical model}

In our numerical model of a rock joint, six plates delimit a deformable parallelepiped box representing filling material of the rock joint between two adjacent blocks: the upper plate of the box represents the in-contact surface of the upper block (its lower side), whereas the bottom plate of the box represents the in-contact surface of the lower block (its upper side) (see Figure 1). This parellelepiped box is filled with discrete elements, which represent the gouge material filling the rock joint. Deformations of the box simulate the relative movements of the two adjacent blocks.

The stresses and relative displacements which describe the mechanical state of the rock joint are deduced, respectively, from the forces acting on the upper plate of the box and from its displacements. By defining $\overrightarrow{F_{\text {sup }}}$ as the force sustained by the upper side, $S$ as its surface in contact with the discrete elements, and $Y_{c}$ and $X_{c}$ as the current coordinates of its centre, we obtain:

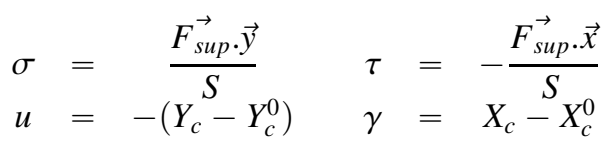

$X_{c}^{0}$ and $Y_{c}^{0}$ are the initial values of $Y_{c}$ and $X_{c}$, which will be defined below. A "-" sign is chosen in the definition of $\tau$ to obtain positive values of $\tau$ and positive values of $\gamma$ during shear loading (for the sake of clarity of the curves). The other "-" sign of the definition of $u$ corresponds to the conventional choice of having positive values in compression.

Before going into the details of the simulations, let us note that gravity is ignored in this problem. As for the sample's boundaries, the surrounding vertical plates (with 
normal directed by $\vec{x}$ or $\vec{z}$ ) are perfectly non-sliding, whereas the upper and lower ones (with the normal directed by $\vec{y}$ ) have a friction coefficient equal to $\tan \left(37^{\circ}\right)$ : the same as between the spheres inside the sample. This allows to obtain an homogeneous stres field in the sample, which justifies the definition of the mechanical state from the state of the upper side: the previous equation (20).

The box is filled with discrete spherical elements using the packing algorithm developed by Jerier [31]. Spheres of different radii are chosen. Numerical samples of spheres with the same radius have the disadvantage of showing very different mechanical behaviours, depending only on their initial fabric, as Antonellini [32], for example, emphasised. Moreover, crystallisation occurs with such samples. These two phenomena are physically meaningless for rock joint studies and must be avoided. The exact values of the radii are not calibrated on physical specimens; they will be justified by the final results of the model.

In order to obtain a dense sample that will dilate under shear, as rock joints do, an isotropic compression, with an inter-particle friction angle equal to zero, is performed by moving all plates towards the sample. Once a given state is reached, the interparticle friction angle is set to $37^{\circ}$. This value corresponds to the one to be used in the simulations. Moreover, the plates are moved apart in order to cancel any confinement (or to set it to a negligible value). The sample is then considered to be in its virgin state, i.e. $\tau=\sigma=0 \mathrm{kPa}$ and $u=\gamma=0 \mathrm{~m}$ (this is how $X_{c}^{0}$ and $Y_{c}^{0}$ from equation (20) are defined), and ready to be loaded in different ways.

\subsection{Simulation of the different loading paths}

Various loading paths can be simulated by the numerical model. Constant normal displacement and constant tangential displacement paths will be considered first because they represent the basic calibration paths, determining the moduli defined in equation (10). Other paths, such as the constant normal load (noted CNL, defined by $\sigma=c s t$ ) or the constant normal stiffness shearings, will also be performed below, in order to validate the constitutive relation defined by equation (13).

\subsubsection{Calibration paths}

The two calibration paths are controlled by applying at each time step:

$-d u=v_{\text {comp }} d t \quad d \gamma=0 \quad$ for the CTD path

$-d u=0 \quad d \gamma=v_{c i s} d t$ for the CND path.

It is verified that $v_{\text {comp }}$ and $v_{\text {cis }}$ are small enough so that the numerical sample remains in quasi-equilibrium over the entire loading paths, which ensures that these two numerical parameters have no influence on the results. In particular, numerical tests always include a "rest" phase when movement of the box is stopped (once a required shear diplacement is reached, for example), in order to verify whether or not, without loading, evolving stresses stop (as they should in quasi-equilibrium).

During the movement of the upper side, Figure 2 shows how the other sides move. The lower one and those in front of or behind the sample (whose normal is $\vec{z}$ ), do not move. The lateral ones are both translated and rotated so that the box always remains closed. 


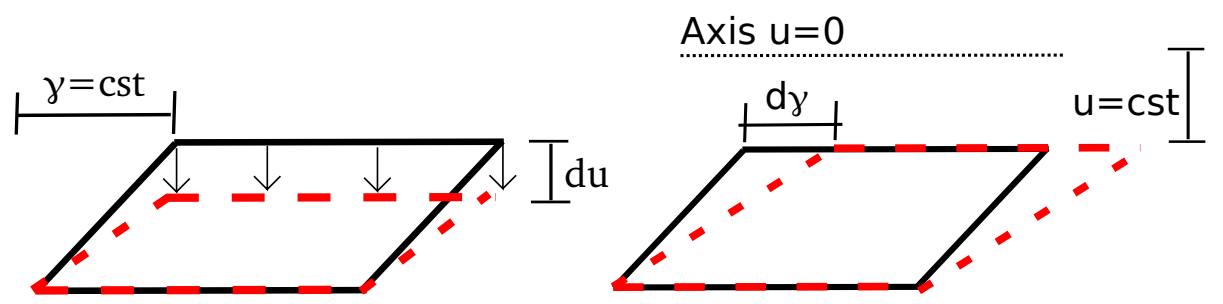

Figure 2: Movements of the box to simulate the CTD $(d \gamma=0)$ path (left) or the CND $(d u=0)$ path (right)

\subsubsection{First validation path: constant normal load shearing (CNL)}

Shearing the numerical sample and maintaining a constant normal stress (i.e. the normal force acting on the top of the box), is straightforward with the discrete element method. When $\sigma$ is different from the desired value of the applied stress $\sigma_{0}$, the stiffness $K_{n}$ of the contact [sample-top plate of the box] $K_{n}$ is determined by adding all stiffnesses of all acting interactions spheres of the [sample-top plate of the box]. Then the top plate is moved in the vertical direction with an amplitude of $d y=\frac{\sigma-\sigma_{0}}{K_{n}}$ in order to obtain $\sigma=\sigma_{0}$. In fact, this is not perfectly true because, for example, this change in $d y$ could correspond to a change in the interpenetrations of $d u_{n}=-d y$ only if the spheres do not move vertically at all, which is never the case in numerical simulations. However, this algorithm allows $\sigma$ to be maintained at a desired value with errors less than $1 \%$.

\subsubsection{Second validation path: constant normal stiffness shearing (CNS)}

This path can be considered an intermediate configuration, in terms of stiffness, between the CNL and the CND paths. It is defined by the application of a shear deformation on the sample, while verifying the following condition,

$$
\forall t \quad \sigma(t)=\sigma_{0}+K_{n} C\left(Y(t)-Y_{0}\right)
$$

In this equation, $K_{n} C$ is the desired normal stiffness value imposed for this loading path. For $K_{n} C=0$, we obtain the CNL conditions, and for $K_{n} C$ increasing towards $\infty$, the loading tends towards the CND conditions. The method used to carry out such tests with the numerical model is exactly the same as the one used to obtain CNL paths, with vertical displacements $d y$ computed so that equation (21) is verified instead of $\sigma(t)=\sigma_{0}$

\section{Results of the discrete element model}

\subsection{Numerical loading paths}

Starting from the virgin state of the sample, the CTD path - a calibration path - is performed in loading and unloading. During all this compression, the tangential stress $\tau$ can be considered equal to zero, whereas Figure 3 represents the changes in the normal stress $\sigma$. The changes in $\tau$ are no longer negligible if such paths are applied from other initial mechanical states, corresponding to increasing shearing (e.g. different values of 
$\gamma)$. The resulting $\sigma(u)$ and $\tau(u)$ curves for such tests are plotted in Figure 4. They will be used to define the moduli of the constitutive relation, since those moduli correspond to the slopes of the curves.

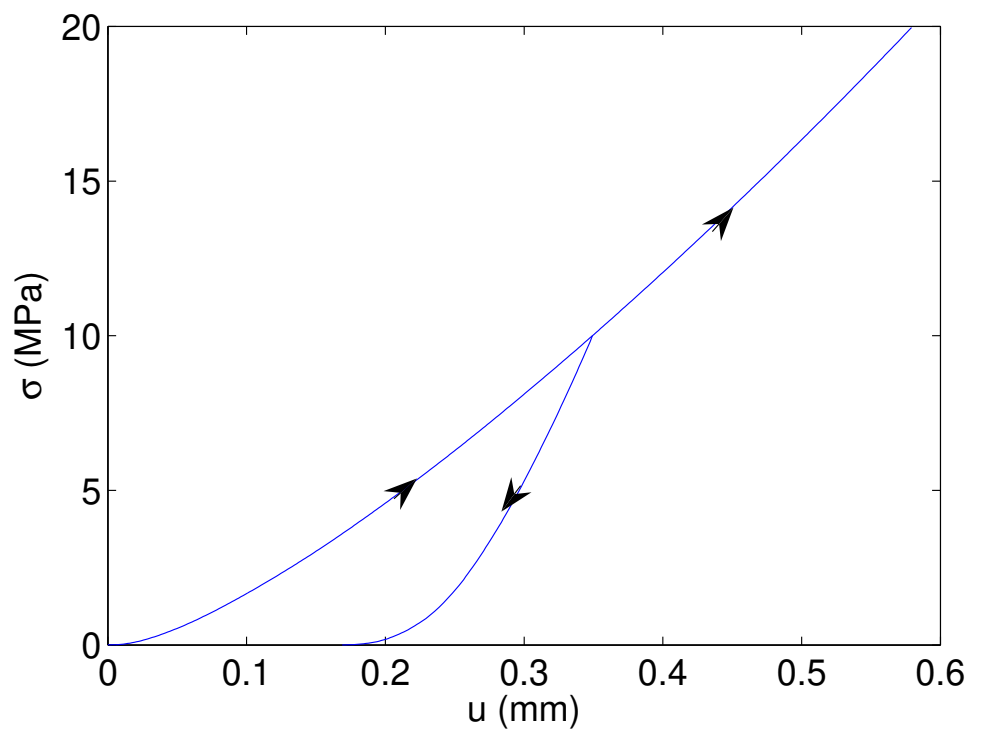

Figure 3: Normal stress versus normal displacement from a simulated CTD test, from the virgin state of the numerical sample

The $\sigma(u)$ (from which $N_{u}^{+}$and $N_{u}^{-}$are defined) curves are nonlinear and show that normal stiffness decreases with the initial shearing level of the sample. Note that these two classical results were previously observed experimentally by Bandis et al. [8] and Saeb and Amadei [10], which shows that the present numerical model is capable of reproducing realistic behaviour. For $\tau(u)$ and its corresponding moduli $G_{u}^{+}$and $G_{u}^{-}$, about which very little information is available in the literature, the discrete model shows an increase of these two moduli with previous shearing.

The results obtained from simulations for the CND calibration path, starting from different initial states defined by different initial normal stresses $\sigma_{0}$ (with $\gamma_{0}=0 \mathrm{~mm}$ ), are presented in Figure 5. As for the changes in $\sigma$ with respect to $\gamma$ (which is described by the moduli $N_{\gamma}^{+}$and $N_{\gamma}^{-}$), we see that the behaviour of the numerical joint contracts first before dilating, as it was observed for infilled discontinuties by Papaliangas et al. [1]. The change is controlled by a given $\frac{\tau}{\sigma}$ ratio, which corresponds to a characteristic frictional angle $\phi_{c}$. Before and after this state, these two moduli, $N_{\gamma}^{+}$and $N_{\gamma}^{-}$, seem to be constant during the shearing process (the related parts of the curves are considered to be linear) and not to depend on the state in compression of the joint. As for the $\tau(\gamma)$ curve (thus the $G_{\gamma}^{+}$and $G_{\gamma}^{-}$moduli), $G_{\gamma}^{-}$could probably be considered constant, whereas it seems to be convenient to let $G_{\gamma}^{+}$evolve during shearing to take into account the material hardening (the exact definitions of the moduli will be detailed hereafter). 

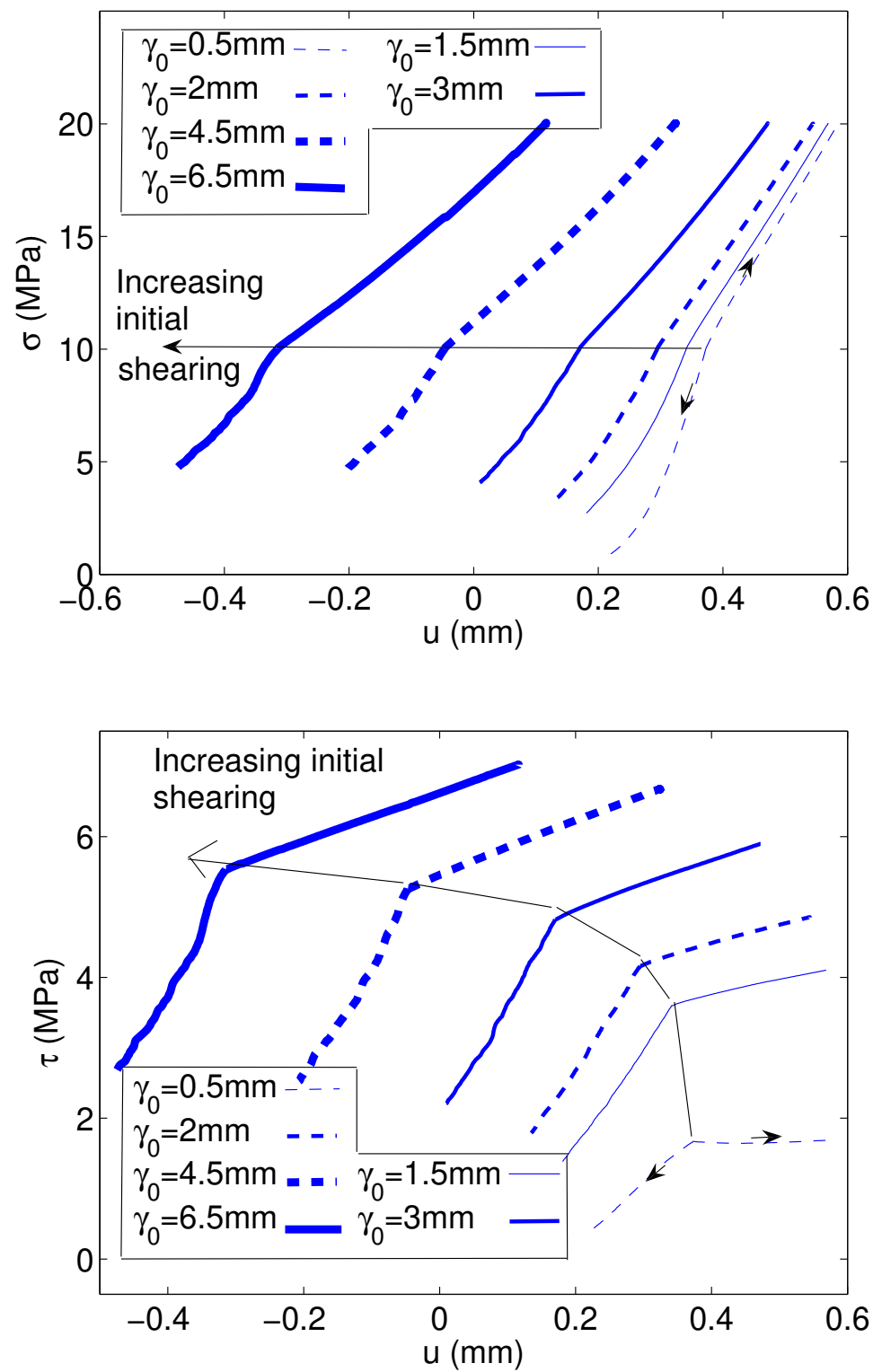

Figure 4: Stresses versus normal displacement curves resulting from simulated CTD tests, from sheared states of the numerical sample 

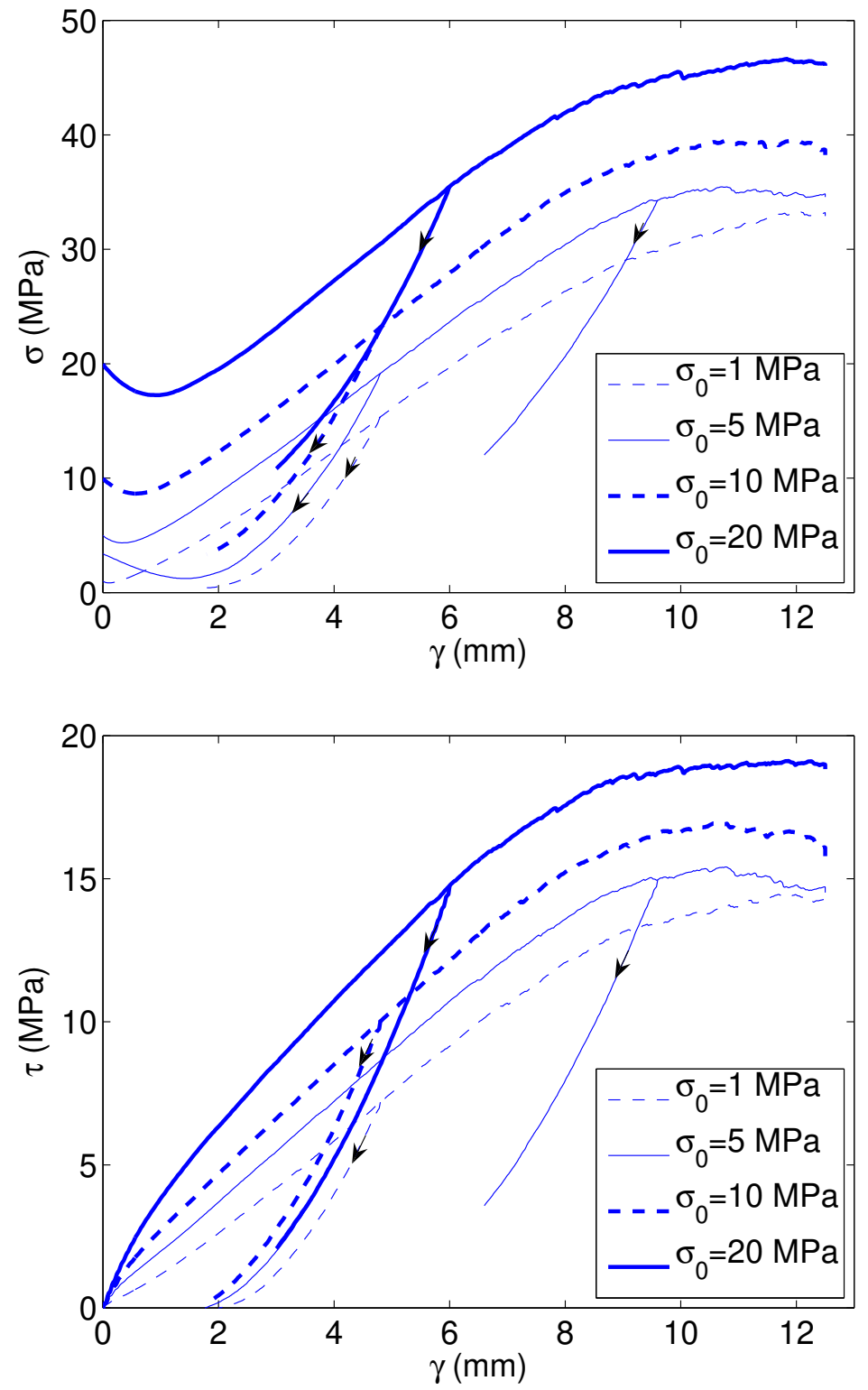

Figure 5: Stresses versus tangential displacement curves resulting from simulated CND tests. For $u_{0}=$ $0.07 ; 0.21 ; 0.35 ; 0.58 \mathrm{~mm}$, corresponding to $\sigma_{0}=1 ; 5 ; 10 ; 20 \mathrm{MPa}$ 


\subsection{Taking into account the current shearing state}

It has been shown that some moduli must depend on the current shearing state in order to reproduce a realistic behaviour. One option is to choose these moduli depending on the value of a state variable, such as the tangential relative displacement $\gamma$. This choice has been suggested by the results obtained by Goodman [33] and Leichnitz [9], known as the "constant displacement model". These models give a physical meaning of $\gamma$ when considering its values at peak states or at residual states such as material constants. However, the failure criterion, such as the Mohr-Coulomb criterion used here, does not depend on a displacement variable. This is why another choice of state variable is proposed by considering $\tau / \sigma$ as the state variable which will control the moduli.

Two sets of pairs ( $G_{u}^{-}$versus $\gamma$ or $\left.\tau / \sigma\right)$ are considered (see Figures 6 and 7). For the first set, the values of $G_{u}^{-}$are computed from unloading œdometric compressions performed immediately after a CNL path.

For the second one, a CND shear, then a loading œdometric compression, are performed before the unloading œdometric compression so that $G_{u}^{-}$can be computed.

This leads to different $\tau / \sigma$ ratios for the same value of $\gamma$, and Figure 6 shows that this impacts the values of $G_{u}^{-}$determined from the numerical simulations. On the other hand, Figure 7 shows that plots according to $\tau / \sigma$ seem to unify all the results so that $\tau / \sigma$ is indeed the right variable to represent shearing. This conclusion tends to disagree with the "constant displacement models" of [33] and [9].

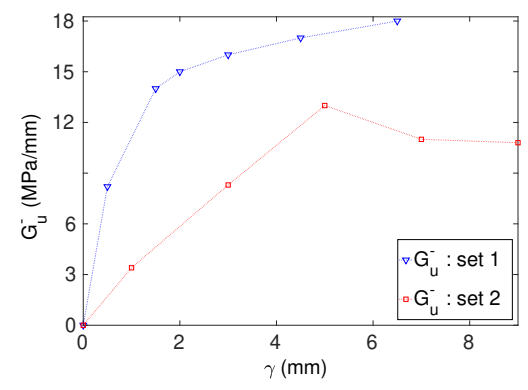

Figure 6: $G_{u}^{-}$versus $\gamma$

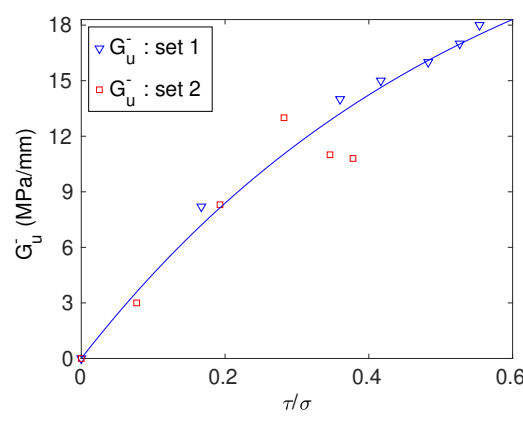

Figure 7: $G_{u}^{-}$versus $\frac{\tau}{\sigma}$. A single master curve appears

\subsection{Determination of a macroscopic friction angle}

When using the $\tau / \sigma$ ratio in the constitutive relation, its variation range needs to be set up. As was suggested by the experimental results of Papaliangas et al. [1], we assume that a Mohr-Coulomb criterion, with zero cohesion, can be used to describe the domain of $(\tau ; \sigma)$ values that can be endured by the infilled joint.

To determine the value of the corresponding macroscopic friction angle $\phi$, it is possible to see how the mobilised friction angle $\phi_{m}$ (defined by $\tan \left(\phi_{m}\right)=\tau / \sigma$ ) evolves during five different numerical tests (see Figure 8). This figure considers two constant 
normal stress shearings (at two normal different stresses), two constant normal displacement shearings (at two different initial stresses), and one œdometric unloading, from a completely sheared initial state (which is the state of the numerical sample at the end of one of the constant normal stress shearings).

Because the macroscopic maximum value of $\phi_{m}$ should be equal to $\phi$, we conclude from Figure 8 that our numerical model of a rock joint has a friction angle of around $29^{\circ}$. Moreover, one can observe first that the CND shearing in fact does not reach the Mohr-Coulomb criterion exactly (their maximum value of $\phi_{m}$ is smaller than $\phi$ ). Second, the results also reveal that once the sample has reached the Mohr-Coulomb criterion after one CNL shearing, it follows this criterion downwards during the CTD (œdometric) unloading: $\tau$ decreases whereas $\tan \left(\phi_{m}\right)=\tau / \sigma$ remains constant and equal to $\tan \left(29^{\circ}\right)$.

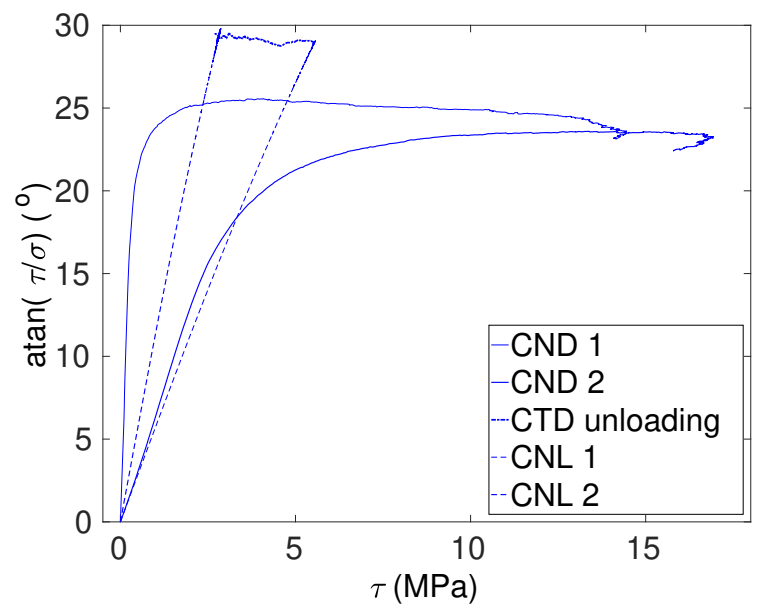

Figure 8: Determination of a macroscopic friction angle: changes of the mobilised friction angle during numerical tests

\subsection{Experimental results}

Before achieving the calibration process of the constitutive relation from the numerical model, let us present experimental data, which confirm the relevancy of the discrete model used to reproduce the behaviour of the infilled rock joints. Some quantitative comparisons will be presented, but we will focus here on qualitative comparisons: we aim at checking if the trends shown by our numerical model (especially the link between $d \tau$ and $d u$ ) correspond to a real behaviour. Because our main goal is to define a constitutive relation relying on these observed trends, we lay stress on qualitative checks for this first step. Quantitative calibration will be a future part. This shows the advantages of the numerical model, which can give exhaustive and less scattered results.

These experiments were conducted by partners of the French LRPC (Laboratoire Régional des Ponts et Chaussées) of Toulouse on natural specimens of gneiss from 
the Gorges de Valabres site located in southern France. Figure 9 shows the state of one sample after one shearing: the presence of an infill material in these specimens is obvious. Experimentally, one CND shear was carried out, as well as œdometric

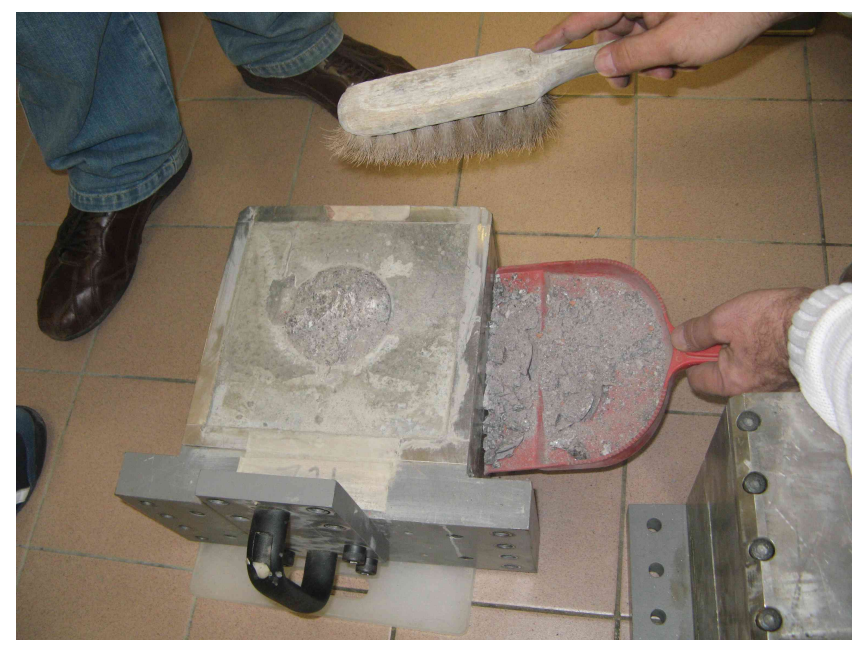

Figure 9: Infill material in one specimen of the tested rock joints

compressions.

\subsubsection{An experimental CND path}

It was emphasised in Section 1 that CND paths are very difficult to follow with rock joints. One must remain critical when considering these experimental results. Figure 10 verifies whether the experimental loading path succeeded in preventing all normal displacements.

The relative normal displacement $u$ obtained during the experiment is plotted for four shear paths: three CNL paths at different stresses and the CND path. This relative normal displacement is obtained by considering the mean of four sensor measurements located at each corner of the shear box. It can be observed that in this experimental CND path the values of $u$ are not strictly null; however, their values remain low compared to other shear loading tests.

Let us now consider Figure 11 where the stresses obtained during this experimental test are plotted. The left part shows the whole curves and it appears that the curves, $\sigma(\gamma)$ especially, are similar to those obtained from the discrete numerical model (see Figure 5), noting that smaller stresses are reached experimentally than those obtained by the numerical model. On the right part of Figure 11, a zoom on the beginning of the curves is represented. This zoom allows one to compute the values of moduli $N_{\gamma}^{+}$ and $G_{\gamma}^{+}$corresponding to this experiment. A value between 2 and $4 \mathrm{MPa} / \mathrm{mm}$, which is very similar to the 3.4 from the numerical model, has been evaluated for $N_{\gamma}^{+}$dil. For $G_{\gamma}^{+}$, the initial values of the slopes indicate a value around $2 \mathrm{MPa} / \mathrm{mm}$, which is here again similar to the values deduced numerically, ranging from 1.2 to 3.8 . 


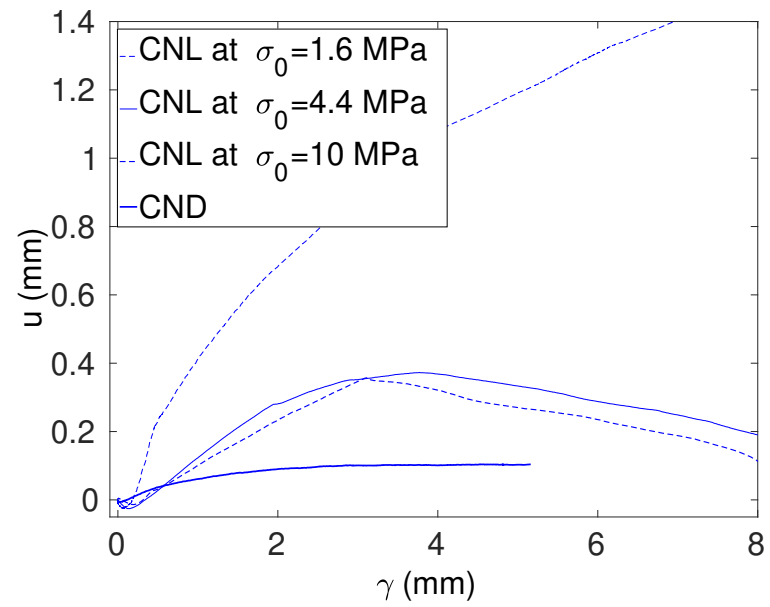

Figure 10: Dilation obtained experimentally for various shear paths, including the CND calibration path
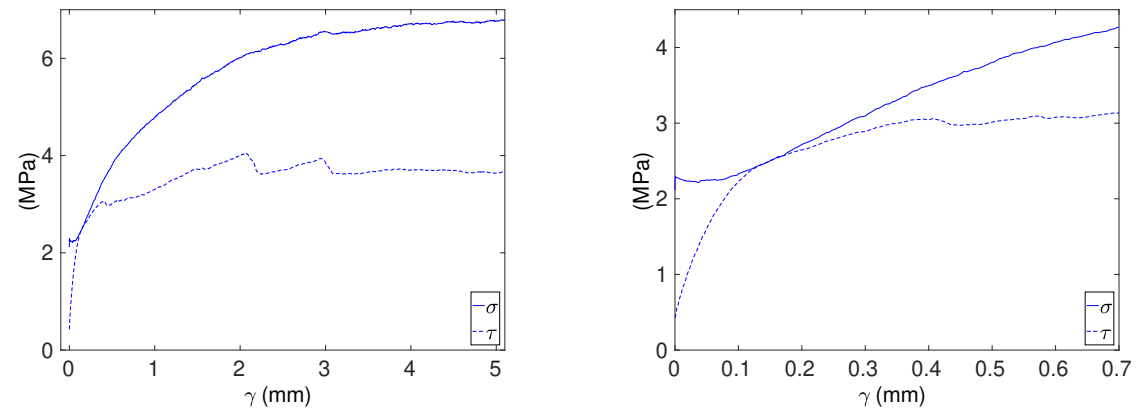

Figure 11: Whole (left) or zoomed (right) experimental responses of a rock joint along a CND path 


\subsubsection{Experimental CTD paths}

A number of œdometric compression tests were also carried out. The same sample was brought under a constant normal stress of $0.5 \mathrm{MPa}$ to different values of $\gamma$. Then different CTD paths were performed using loading and unloading steps. Figure 12 shows resulting $\sigma(u)$ curves. Experimental results illustrate the decrease in normal stiffness due to shearing, which was mentionned in paragraph 3.1. The first curve, with $\gamma_{0}=0 \mathrm{~mm}$, is an exception, because of the experimental setting up. A numerical curve, issued from the discrete model, shows that the normal stiffness of our model, in a non-sheared state, is close to the one experimentally measured.

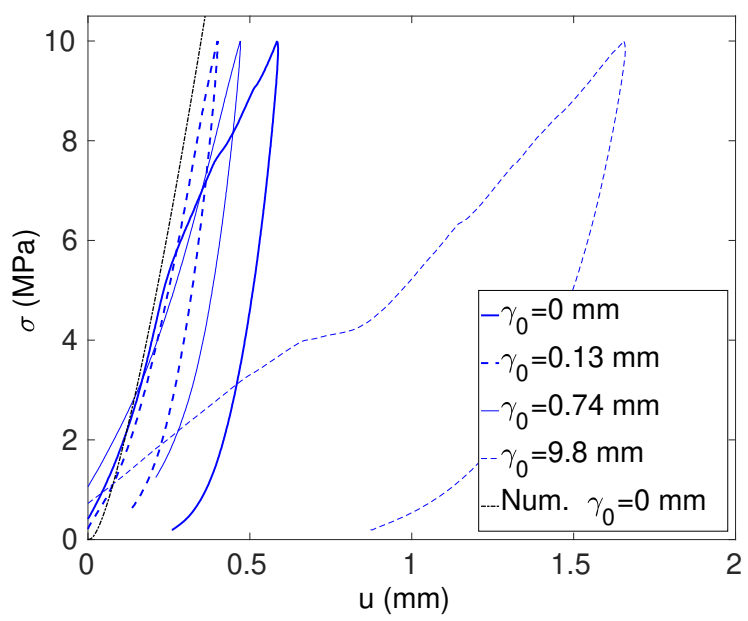

Figure 12: Experimental and numerical ("num.") $\sigma(u)$ curves for CTD paths

Then, because of the lack of knowledge on the changes in $\tau$ during these experiments, particular attention is paid to the changes in $\tau$ depending on $u$ (see Figure 13). Because of the dilatancy occuring during the CNL shear loading, the initial values of $u$ at the beginning on each CTD path are in fact different; therefore, to clarify the figure, the values are set to zero for each compression test.

Except the curve for $\gamma=9.8 \mathrm{~mm}$, it appears that the slopes of the experimental $\tau(u)$ curves, which define the moduli $G_{u}^{+}$and $G_{u}^{-}$, increase when a previous shearing loading has been applied to the sample, i.e. when $\gamma_{0}$ increases. This was shown by the numerical discrete element model and is now experimentally confirmed. Moreover, it should be noted that this sample exhibits a peak for curve $\tau(\gamma)$ for CNL shear, and that the last value of $\gamma_{0}$ considered corresponds to the post-peak region. The fact that the curve corresponding to this $\gamma_{0}$ does not have the higher slope value confirms the choice of the variable $\tau / \sigma$, instead of $\gamma$, to describe the influence of the previous shearing path on the moduli. This curve presents a $\tau / \sigma$ ratio lower than the ratio determined on the curve at $\gamma=0.74 \mathrm{~mm}$, and it confirms that describing the influence of shearing through $\tau / \sigma$ explains why the slopes are smaller, even if $\gamma_{0}$ is higher. 


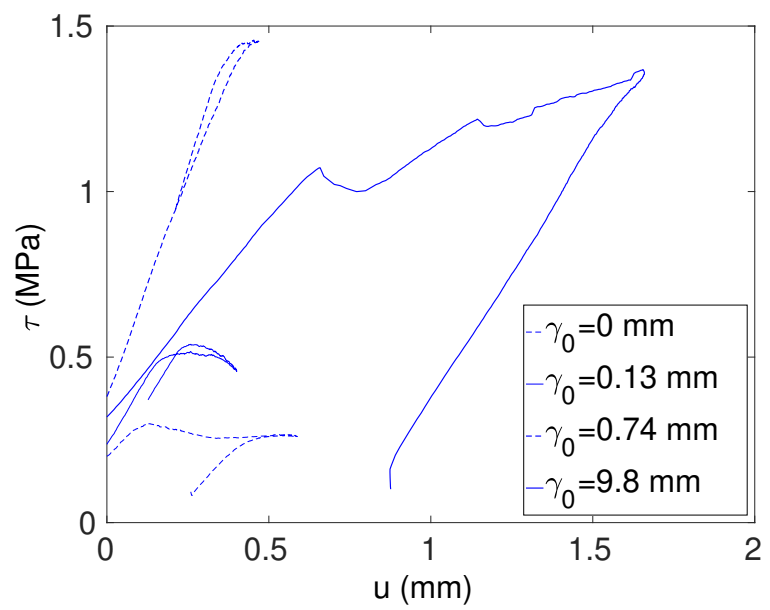

Figure 13: Experimental $\tau(u)$ curves for CTD paths performed on the same sample

\section{Calibration of the constitutive relation}

The moduli will now be clearly defined and we will verify that their definition reproduces the behaviour of the numerical model for the calibration paths, i.e. that our constitutive relation is well calibrated.

\subsection{Definition of the moduli}

Considering all remarks formulated in previous sub-sections, we define the values for the moduli in equations (22) and (23):

$$
\begin{aligned}
& N_{\gamma}^{+}=\left\{\begin{array}{cc}
-N_{\gamma}^{0} & \text { if } \tau / \sigma<\tan \left(\phi_{c}\right) \\
N_{\gamma}^{+} \text {dil } & \text { if } \tan \left(\phi_{c}\right) \leqslant \tau / \sigma<\tan (\phi) \\
0 & \text { if } \tau / \sigma=\tan (\phi)
\end{array}\right. \\
& N_{\gamma}^{-}=\left\{\begin{array}{cc}
-N_{\gamma}^{0} & \text { if } \tau / \sigma<\tan \left(\phi_{c}\right) \\
3.5 N_{\gamma}^{+} \text {dil } & \text { if } \tan \left(\phi_{c}\right) \leqslant \tau / \sigma \leqslant \tan (\phi)
\end{array}\right. \\
& G_{\gamma}^{+}=\left\{\begin{array}{cl}
G_{\gamma}^{0}-\left(G_{\gamma}^{0}-G_{\gamma}^{f}\right) \frac{1-e^{-\frac{\tau / \sigma}{\tan (\phi)}}}{1-e^{-1}} & \text { if } \tau / \sigma<\tan (\phi) \\
0 & \text { if } \tau / \sigma=\tan (\phi)
\end{array}\right. \\
& G_{\gamma}^{-}=G_{\gamma}^{0}
\end{aligned}
$$




$$
\begin{aligned}
& N_{u}^{+}=N\left(\frac{\tau}{\sigma}\right)\left(\frac{\sigma}{\sigma_{0}}\right)^{\frac{1}{3}} ; \quad N_{u}^{-}= \\
& G_{u}^{+}=G_{u \max }^{+} \frac{1-e^{-\frac{\tau / \sigma}{\tan (\phi)}}}{1-e^{-1}} ; \quad G_{u}^{-}=G_{u \max }^{-} \frac{1-e^{-\frac{\tau / \sigma}{\tan (\phi)}}}{1-e^{-1}}
\end{aligned}
$$

The $N\left(\frac{\tau}{\sigma}\right)$ function which describes the decrease in normal stiffness of the rock joint due to shearing is a power function, defined by:

$$
N\left(\frac{\tau}{\sigma}\right)=\frac{20 N_{u}^{0}-N_{u}^{f}}{19}-\frac{N_{u}^{0}-N_{u}^{f}}{19} 20^{\frac{\tau / \sigma}{\tan (\phi)}}
$$

This expression corresponds to a decrease in normal stiffness, which is increasingly important while shearing takes place (the derivative of $N\left(\frac{\tau}{\sigma}\right)$ increases with $\frac{\tau}{\sigma}$ ), as the numerical simulations have shown.

The parameters introduced in the previous equations are summarized in Table 2, with the retained values.

Table 2: Parameters of the constitutive relation

\begin{tabular}{|c|c|c|c|c|c|c|c|c|c|c|}
\multicolumn{9}{|c|}{$(\mathrm{MPa} / \mathrm{mm})$} & $(\mathrm{MPa})$ & \multicolumn{2}{c|}{$(\mathrm{MPa} / \mathrm{mm})$} & \multicolumn{2}{|c|}{0} \\
$N_{\gamma}^{0}$ & $N_{\gamma \text { dil }}^{+}$ & $G_{\gamma}^{0}$ & $G_{\gamma}^{f}$ & $N_{u}^{0}$ & $N_{u}^{f}$ & $\sigma_{0}$ & $G_{u}^{+}$max & $G_{u \text { max }}^{-}$ & $\phi$ & $\phi_{c}$ \\
\hline 2.4 & 3.4 & 3.8 & 1.2 & 20 & 8 & 1 & 3.7 & 17.5 & 29 & 12
\end{tabular}

$N_{\gamma}^{0}$ is the opposite of the slope (supposed to be constant) of the contractant part of the $\sigma(\gamma)$ curve measured during a CND path: the higher its value is, the more contractant the joint is. $N_{\gamma}^{+}$il is similar but concerns the dilatant part of the same curve. The parameter $\phi_{c}$ governs the contractant-dilatant transition, which occurs for $\tau / \sigma=\tan \left(\phi_{c}\right)$.

$G_{\gamma}^{0}$ and $G_{\gamma}^{f}$ correspond respectively to the initial or final tangential rigidity - the slope of curve $\tau(\gamma)$ - during the same CND path. $G_{\gamma}^{f}$ is reached for $\tau / \sigma=\tan (\phi)$, with $\phi$ an other parameter which corresponds to a macroscopic friction angle.

$N_{u}^{0}$ corresponds to the normal rigidity during a CTD path in load, under a value of $\sigma$ equal to $\sigma_{0}$ and without previous shearing: $\tau=0$; while $N_{u}^{f}$ is obtained when the joint is completely sheared $\tau=\tau_{\max }=\sigma \tan (\phi)$.

$G_{u \text { max }}^{+}$and $G_{u \text { max }}^{-}$are the slopes of $\tau(u)$ curves along CTD paths, under complete shearing: $\tau / \sigma=\tan (\phi)$, for $d u>0$ or $d u<0$ respectively. All these parameters are obtained through trial and error attempts, by approximating non-linear curves by linear ones when needed (for $N_{\gamma}^{0}$ or $N_{\gamma}^{+}$dil for example). 


\subsection{Comparisons between the constitutive relation and the numerical model along the calibration paths}

Let us first verify that these expressions of the moduli presented in equations (22) and (23) are satisfactory according to the numerical model and can indeed reproduce its behaviour along the calibration paths. Figures 14 and 15 compare the responses of the numerical model and the responses of the constitutive relation along these paths.
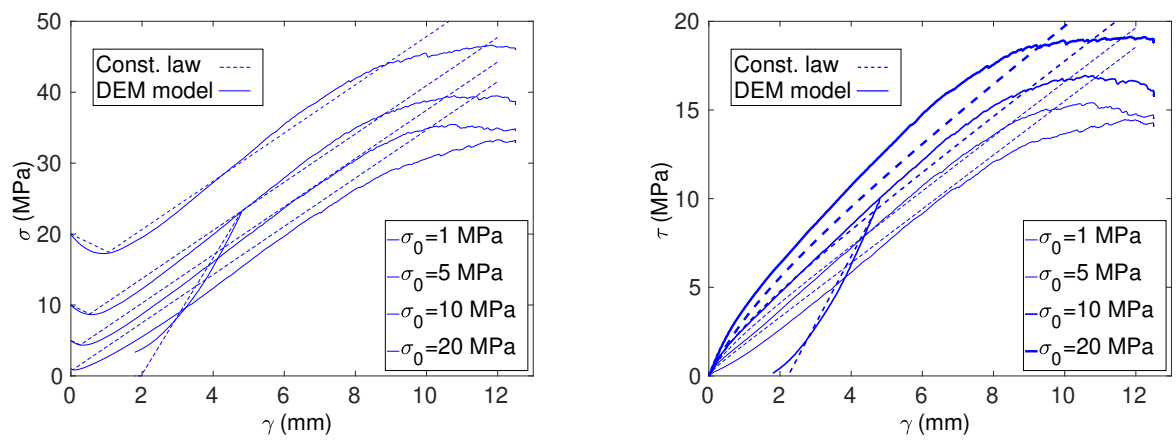

Figure 14: Responses of the numerical model (solid lines) and the constitutive relation (dotted lines) along the CND calibration path
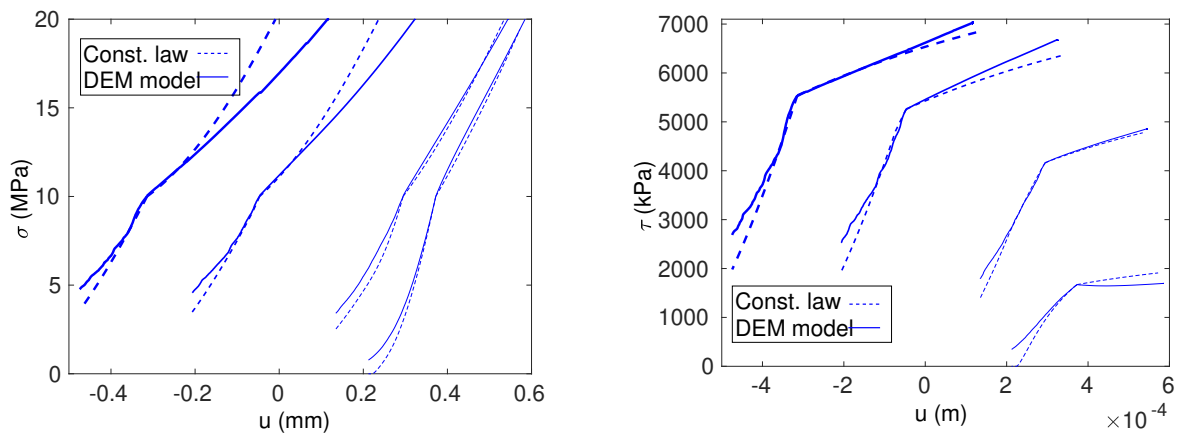

Figure 15: Responses of the numerical model (solid lines) and the constitutive relation (dotted lines) along the CTD calibration path

These two figures show good agreement and prove that the constitutive relation is properly calibrated.

\section{Validation of the constitutive relation by considering other loading paths}

The relation can now be validated using comparisons along the validation paths: paths other than the calibration paths. Other loading paths were simulated with the numerical discrete element model in order to validate the constitutive relation by checking if equation (13) is able to predict the behaviour of the numerical model. Four CNL and 
three constant normal stiffness shearings were carried out. The responses are compared with predictions of the constitutive relation established in equation (13) (see following sections and Figures 16 and 17).

\subsection{Validation for CNL paths}

Four numerical CNL shear paths were so carried out for different initial stresses $\sigma_{0}$. Figure 16 shows the comparison between the constitutive relation and the numerical model on these paths and validates the relation quite well. Let us note that the results (of the numerical model or of the constitutive relation) do not present any peak of the tangential stress on these loading paths. This lack of peak was observed experimentally by Papaliangas et al. [1] for discontinuities presenting a given filler thickness.
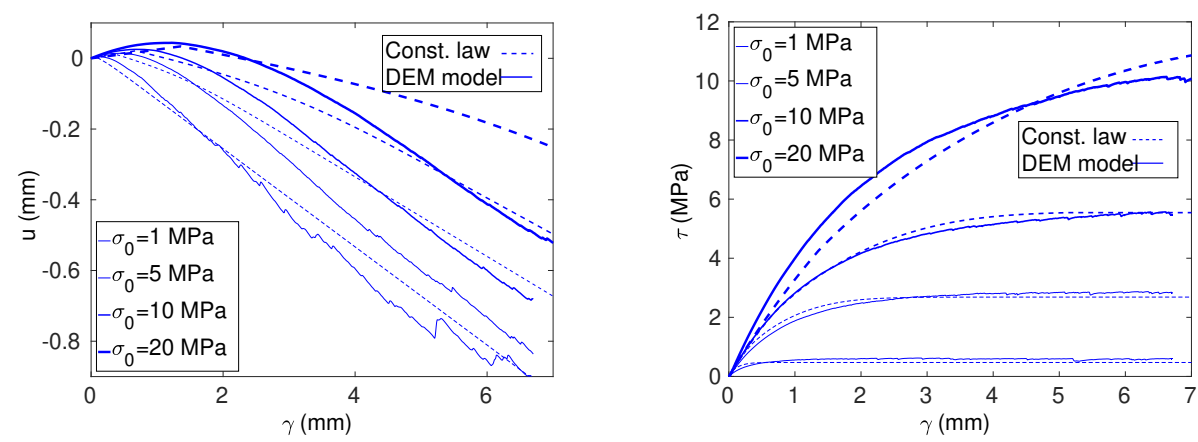

Figure 16: Responses of the numerical model and the constitutive relation along the CNL paths: $u(\gamma)$ (left) and $\tau(\gamma)$ (right) curves

\subsection{Validation for CNS paths}

The constant normal stiffness paths are now considered. Three CNS shearing tests using the same initial normal stress, $\sigma_{0}=5 \mathrm{MPa}$, but three different rigidity moduli, $K n C$, were performed with the numerical model. Their respective results were compared to the constitutive relation (see Figure 17). Here again the predictive capacities of the constitutive relation are verified.

\section{Conclusion}

Using the results of a numerical discrete model of an infilled rock joint, complemented by experimental data, a constitutive relation has been built, calibrated and validated. Because of the incrementally nonlinear nature of the relation, it has been possible to avoid introduction of yield surfaces and flow rules, which are difficult to determine experimentally in the case of rock joints. Irreversibilities due to the plastic strain are described directly (i.e. without considering any loading-unloading criterion) through incremental nonlinearity. The method to build the relation was deduced from the general incremental nonlinear formalism proposed by Darve and Labanieh [12] and Darve et al. [34]. 


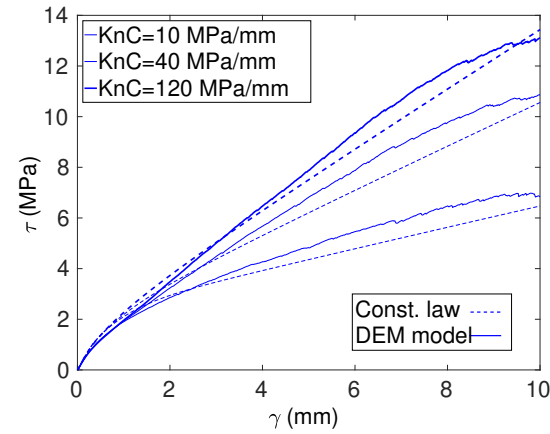

(a) $\tau(\gamma)$

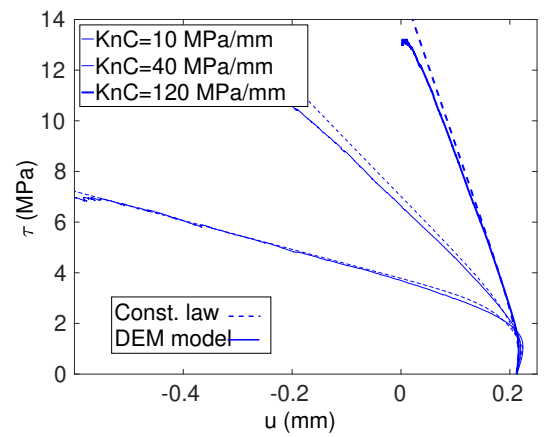

(c) $\tau(u)$

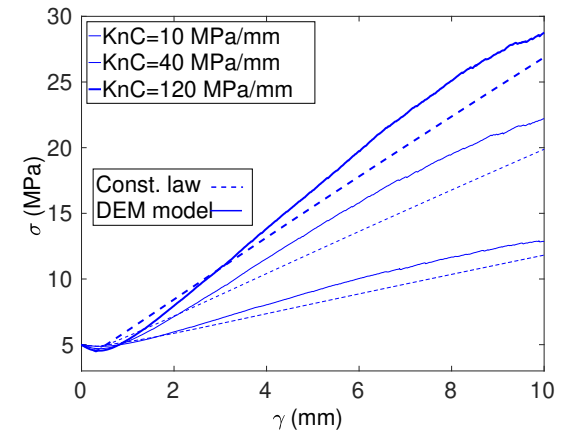

(b) $\sigma(\gamma)$

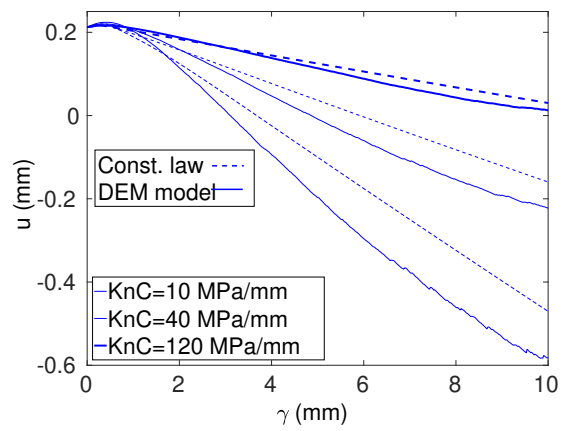

(d) $u(\gamma)$

Figure 17: Comparison between predictions of the discrete model (solid lines) or the constitutive relation (dotted lines) for CNS paths 
The definition of this relation is very general and it can describe the effects of the normal relative displacement variations on the tangential stress, acting along rock joints. Numerical and experimental tests have shown that such effects are crucial for joint behaviour, especially when the joint is increasingly sheared. Moreover, this shearing process, which also influences the value of the normal stiffness, is properly simulated when the $\tau / \sigma$ ratio is used as a state variable, instead of the tangential displacement $\gamma$.

Finally, we have shown that this constitutive relation is capable of predicting the response of the numerical rock joint along various paths, such as constant normal loads as well as constant normal stiffnesses. Implemented in UDEC [35] - a discrete software

using a "block system" approach - it has been used to compute the stability of actual cliffs.

\section{Acknowledgements}

The authors wish to thank the members of the French STABROCK research programme, particularly Muriel Gasc and other researchers from LRPC, Toulouse, for having conducted the experiments.

[1] T. Papaliangas, S. R. Hencher, A. C. Lumsden, and S. Manolopoulou, "The effect of frictional fill thickness on the shear strength of rock discontinuities," Int. J. Rock Mech. Min. Sci. E Geomech. Abstr., vol. 30, pp. 81-91, 1993.

[2] J. P. Pereira, "Rolling friction and shear behaviour of rock discontinuities filled with sand," Int. J. Rock Mech. E Min. Sci., vol. 34, pp. 244e1-244e17, 1997.

[3] H. K. Kutter and A. Raunterberg, "The residual shear strength of filled joints in rock," in Proc. 4th Symp. ISRM, pp. 221-227, 1979.

[4] R. D. Lama, "Influence of clay fillings in shear behaviour of joints," in Proc. 3rd Int. Conf. Int. Association of Engineering Geology, pp. 27-34, 1978.

[5] B. Indraratna and A. Haque, "Experimental study of shear behavior of rock joints under constant normal stiffness conditions," Int. J. Rock Mech. E Min. Sci., vol. 34, no. 3-4, 1997.

[6] M. Boulon, G. Armand, N. Hoteit, and P. Divoux, "Experimental investigations and modelling of shearing of calcite healed discontinuities of granodiorite under typical stresses," Engineering Geology, vol. 64, pp. 117-133, 2002.

[7] Goodman, Taylor, and Brekke, "A model for the mechanics of jointed rock," Journal of the soil mechanics and Foundation division ASCE, vol. 14, pp. 637-659, 1968.

[8] S. C. Bandis, A. C. Lumsden, and N. R. Barton, "Fundamentals of rock joint deformation," Int. J. Rock Mech. Min. Sci. E Geomech. Abstr., vol. 20, no. 6, pp. 249-268, 1983. 
[9] W. Leichnitz, "Mechanical properties of rock joints," International Journal of Rock Mechanics and Mining Sciences $\mathcal{E}$ Geomechanics Abstracts, vol. 22, no. 5, pp. $313-321,1985$.

[10] S. Saeb and B. Amadei, "Modelling rock joints under shear and normal loading," Int. J. Rock Mech. Min. Sci. E Geomech. Abstr., vol. 29, no. 3, pp. 267-278, 1992.

[11] M. E. Plesha, "Constitutive models for rock discontinuities with dilatancy and surface degradation," International Journal for Numerical and Analytical Methods in Geomechanics, vol. 11, pp. 345-362, 1987.

[12] F. Darve and S. Labanieh, "Incremental constitutive law for sands and clays. simulations of monotonic and cyclic tests," International Journal for Numerical and Analytical Methods in Geomechanics, vol. 6, pp. 243-275, 1982.

[13] A. S. Saada and G. Bianchini, "Constitutive equations for granular non-cohesive soils," in International Workshop on Constitutive Equations for Granular Noncohesive Soils (1987: Cleveland, Ohio), AA Balkema, 1989.

[14] P. Cundall and O. Strack, "A discrete numerical model for granular assemblies," Géotechnique, vol. 29, pp. 47-65, 1979.

[15] P. Kulatilake, B. Malama, and J. Wang, "Physical and particle flow modeling of jointed rock block behavior under uniaxial loading," International Journal of Rock Mechanics and Mining Sciences, vol. 38, no. 5, pp. 641 - 657, 2001.

[16] J. Wang, Y. Ichikawab, and C. Leung, "A constitutive model for rock interfaces and joints," Int. J. Rock Mech. E Min. Sci., vol. 40, pp. 41-53, 2003.

[17] J.-W. Park and J.-J. Song, "Numerical simulation of a direct shear test on a rock joint using a bonded-particle model," Int. J. Rock Mech. E Min. Sci., vol. 46, pp. 1315-1328, 2009.

[18] M. Jean and J.-J. Moreau, "Unilaterality and granular friction in the dynamics of rigid body collections," in Proc. of the Contact Mechanics Int. Symp. (A. Curnier, ed.), pp. 31-48, 1992.

[19] F. Radjai, D. E. Wolf, M. Jean, and J.-J. Moreau, "Bimodal character of stress transmission in granular packings," Physical Review Letters, vol. 80, no. 1, pp. 61-64, 1998.

[20] B. Cambou and M. Jean, Micromécanique des milieux granulaires. Hermes Science, 2001.

[21] F.-V. Donzé, V. Richefeu, and S.-A. Magnier, "Advances in discrete element method applied to soil, rock and concrete mechanics," Electronic Journal of Geotechnical Engineering, vol. Special Volume, pp. 1-44, 2009.

[22] L. Sibille, F.-V. Donzé, F. Nicot, B. Chareyre, and F. Darve, "From bifurcation to failure in a granular material: a dem analysis," Acta Geotechnica, vol. 3, no. 1, pp. 15-24, 2008. 
[23] N. Barton, "The shear strength of rock and rock joints," Int. J. Rock Mech. Min. Sci. E Geomech. Abstr., vol. 13, pp. 255-279, 1976.

[24] F. Calvetti, G. Viggiani, and C. Tamagnini, "A numerical investigation of the incremental behavior of granular soils," Rivista Italiana di Geotecnica, vol. 3, pp. 11-29, 2003.

[25] N. Belheine, J. Plassiard, F. Donzé, F. Darve, and A. Seridi, "Numerical simulation of drained triaxial test using 3d discrete element modeling," Computers and Geotechnics, vol. 36, no. 1-2, pp. 320-321, 2009.

[26] V. Smilauer, E. Catalano, B. Chareyre, S. Dorofeenko, J. Duriez, A. Gladky, J. Kozicki, C. Modenese, L. Scholtès, L. Sibille, J. Stránský, and K. Thoeni, Yade Documentation. The Yade Project, 1st ed., 2010. http://yade-dem. org.

[27] J. Kozicki and F.-V. Donzé, "YADE-OPEN DEM: an open-source software using a discrete element method to simulate granular material," Engineering Computations, vol. 26, no. 7, pp. 786-805, 2009.

[28] K. Iwashita and M. Oda, "Rolling resistance at contacts in simulation of shear band development by DEM," Journal of Engineering Mechanics, vol. 124, no. 3 , 1998.

[29] J.-P. Plassiard, N. Belheine, and F.-V. Donzé, "A spherical discrete element model : calibration procedure and incremental response," Granular Matter, vol. 11, 2009.

[30] P. Cundall, Computer simulations of dense sphere assemblies, vol. 20 of Studies in Applied Mechanics, pp. 113-123. Elsevier Science, 1988.

[31] J.-F. Jerier, D. Imbault, F.-V. Donzé, and P. Doremus, "A geometric algorithm based on tetrahedral meshes to generate a dense ploydisperse sphere packing," Granular Matter, vol. 11, 2009.

[32] M. A. Antonellini and D. P. Pollard, "Distinct element modeling of deformation bands in sandstone," Journal of Structural Geology, vol. 17, no. 8, pp. 1165-1182, 1995.

[33] R. E. Goodman and W. Boyle, "Non-linear analysis for calculating the support of a rock block with dilatant joint faces," in 34th Geomechanics Colloquy, 1985.

[34] F. Darve, E. Flavigny, and M. Meghachou, "Yield surfaces and principle of superposition: Revisit through incrementally non-linear constitutive relations," International Journal of Plasticity, vol. 11, no. 8, pp. 927-948, 1995.

[35] Itasca, UDEC Version 4.0: Use Manual, 2007. 